\title{
DESENVOLVIMENTO DE UMA FORMULAÇÃO COSMECÊUTICA PARA REGIÃO DOS OLHOS COM EXTRATO PADRONIZADO DE CAFÉ VERDE
}

\section{DEVELOPMENT OF A COSMECEUTIC FORMULATION FOR THE EYES WITH GREEN COFFEE EXTRACT STANDARD}

\author{
Ana Carolina Forgati dos SANTOS', Daneysa Lahis KALSCHNE², Marcelo Caldeira \\ VIEGAS ${ }^{3}$, Lucimara Salvat VANINI ${ }^{4}$, Marta de Toledo BENASSI ${ }^{5}$, Audrey Alessandra \\ Stinghen Garcia LONNI ${ }^{6}$
}

1 - Acadêmica do curso de Farmácia, Universidade Estadual de Londrina, Paraná, Brasil. 2 - Pós-graduação em Ciência de Alimentos, Universidade Estadual de Londrina, Paraná, Brasil. 3 - Companhia Iguaçu de Café Solúvel S.A, Departamento de Pesquisa e Desenvolvimento, Cornélio Procópio-PR, Brasil.

4 - Companhia Iguaçu de Café Solúvel S.A, Departamento de Pesquisa e Desenvolvimento, Cornélio Procópio-PR, Brasil.

5 - Departamento de Ciência e Tecnologia de Alimentos, Universidade Estadual de Londrina, Paraná, Brasil.

6 - Departamento de Ciências Farmacêuticas, Universidade Estadual de Londrina, Paraná, Brasil. Autor correspondente: E-mail: audreylonni@uol.com.br

\section{RESUMO:}

O extrato de café verde da espécie Coffea canephora apresenta significante potencial antioxidante por possuir alto teor de bioativos, como cafeína e ácidos clorogênicos. Radicais livres, associados ao início do processo oxidativo, são um dos principais responsáveis pelo envelhecimento cutâneo. Assim, considerando a importância econômica do café no Brasil e a atual preocupação dos consumidores com a estética, o objetivo do trabalho foi desenvolver e caracterizar uma formulação cosmecêutica, de uso tópico, acrescida de extrato seco liofilizado de café verde para ser utilizada na região dos olhos. Para caracterizar o extrato, foram avaliados o teor de ácidos clorogênicos totais, cafeína e a atividade antioxidante por ABTS. Foram desenvolvidas formulações do tipo emulsão simples (FES) e múltipla (FEM) contendo 0,5\% (p/p) e 1,0\% (p/p) de extrato e avaliadas as características organolépticas, físico-químicas e atividade antioxidante em todas as formulações. Os parâmetros organolépticos e físico-químicos mantiveram-se com valores compatíveis e conforme esperado. As FES apresentaram maior densidade e menor atividade antioxidante que as FEM correspondentes. A FEM0.5 destacou-se pela alta atividade antioxidante $(3,89 \mathrm{~g}$ Trolox $/ 100 \mathrm{~g})$, sugerindo-se o uso na região dos olhos como potencial preventivo do envelhecimento cutâneo, entretanto, mais estudos são necessários para verificar a sua eficácia e segurança.

PALAVRAS CHAVE: Coffea canephora; Atividade antioxidante; Envelhecimento cutâneo.

\section{ABSTRACT:}

The extract of green coffee of the Coffea canephora species presents significant antioxidant potential related to the high content of bioactive compounds, such as caffeine and chlorogenic acids. Free radicals, associated with the beginning of the oxidative process, are one of the main responsible for skin aging. Considering the economic importance of coffee 
in Brazil, and the current consumer concern with aesthetics, the objective of the research was to develop and characterize a cosmeceutical formulation, topical with the addition of green coffee lyophilized extract to be used in the region of eyes. To characterize the extract, the total chlorogenic acids, caffeine content, and the antioxidant activity by ABTS, were evaluated. Were developed formulation of type simple and multiple emulsion containing 0.5 and $1.0 \%$ of extract and evaluated the organoleptic, the physical and chemical characteristics, and the antioxidant activity by ABTS in all the formulation. The simple emulsion formulation with $0.5 \%$ of extract obtained antioxidant activity of $2.70 \%$, while the simple emulsion formulation with $1.0 \%$ of extract presented $3.44 \%$. In formulations of multiple emulsion with $0.5 \%$ of extract, the antioxidant activity was $3.89 \%$ and for the multiple emulsion with $1.0 \%$ of extract was $4.16 \%$. The organoleptic and chemical physical parameters remained with compatible values and as expected. The multiple emulsion formulation with $0.5 \%$ of extract showed the highest antioxidant activity, so, it is suggested that this formulation can be used in the eyes region as preventive of sking aging, however, more studies are necessary to verify it is efficiency and safety.

KEYWORDS: Coffea canephora; Antioxidant activity; Skin aging.

\section{INTRODUÇÃO}

O processo de envelhecimento altera a estrutura e a função dos órgãos e, no caso da pele, modifica, sobretudo, seu aspecto. O envelhecimento cutâneo pode ser cronológico, quando decorrente do desgaste natural do organismo, ou por foto envelhecimento, devido a fatores ambientais notadamente radiação solar, cuja ação é acumulativa. (HIRATA; SATO; SANTOS, 2004; GANCEVICIENE, et al., 2012).

À medida que a pele envelhece ocorrem modificações como afinamento da derme e diminuição do conteúdo de colágeno, além de outras consequências. Essas alterações são aceleradas pela exposição crônica aos raios ultravioletas, fumo e poluição, gerando a formação de radicais livres. Com isso, há uma elevação no número de lesões oxidativas não reparadas, que alteram o metabolismo e são responsáveis pelo envelhecimento precoce. (HIRATA; SATO; SANTOS, 2004; NICOLAU; COUTO 2007; GANCEVICIENE et al., 2012).

O uso de extratos vegetais em produtos cosméticos tem sido cada vez mais motivado pelos pesquisadores e formuladores da área. Porém, é preciso cautela e um estudo minucioso da eficácia de cosméticos contendo extratos vegetais para a obtenção de produtos de alta qualidade. (POUILLOT et al., 2011; EVANS-JOHNSON et al., 2013; LONNI et al., 2015; DEUSCHLE et al., 2015; AFFONSO et al., 2016).

O Brasil é o maior produtor e líder em exportação de café do mundo, sendo responsável por 30,13\% do mercado internacional (ABIC, 2015). Os produtos de café são atrativos por vários motivos, pois além do agradável sabor e aroma, apresentam potencial atividade antioxidante. A relação entre café e saúde tem sido extensivamente estudada nos 
últimos anos, devido ao forte interesse em alimentos que promovem funções fisiológicas. A capacidade antioxidante do café é decorrente da presença de bioativos naturais do produto, como cafeína e ácidos clorogênicos (ACGs) (VIGNOLI et al., 2016; AFFONSO et al., 2016).

Entre as espécies de café mais comercializadas no mundo, o Coffea canephora é a principal matéria-prima da indústria de café solúvel. Comparativamente ao café arábica, destaca-se em função do maior teor de sólidos e menor preço, e pelo maior teor de cafeína e ACGs (VIGNOLI et al., 2014). Considerando-se a pouca estabilidade térmica dos ACGs ao processo de torra, é interessante a produção de extratos a partir do café não torrado (denominado cru ou verde) (CORSO et al., 2016). Assim, produzindo-se um extrato a partir de café verde, seria possível obter maior concentração de bioativos como cafeína e ACGs e maior atividade antioxidante (CORSO et al., 2016), com valor competitivo e potencial para uso em produtos.

Desta forma, o objetivo do presente trabalho foi desenvolver e caracterizar formulação cosmecêutica, de uso tópico para serem utilizadas na região dos olhos, acrescida de extrato seco de café verde da espécie $C$. canephora, comparando emulsões simples e múltiplas bem como avaliar a atividade antioxidante da formulação.

\section{MATERIAL E MÉTODOS}

\subsection{Preparo do extrato de café verde}

O extrato liofilizado de café verde (ECV) foi elaborado pela Companhia Iguaçu de Café Solúvel (Cornélio Procópio, Paraná, Brasil) utilizando Coffea canephora. O processamento foi feito em planta piloto seguindo processo convencional de extração de café solúvel por percolação em colunas, utilizando a água como solvente de extração. Neste processo, água a $180^{\circ} \mathrm{C}$ foi alimentada no primeiro estágio de percolação (coluna com o café mais antigo) e na sequência percolando os estágios seguintes, até atingir o café mais novo. No último estágio, o extrato encontra o café recém carregado, do qual extrai parte dos sólidos solúveis. Durante o processo, os sólidos solúveis do extrato aumentam, mas a temperatura diminui, então a última coluna contendo café fresco é extraída a uma temperatura próxima a $100^{\circ} \mathrm{C}$ estando o produto sujeito a danos térmicos mínimos. O extrato originado deste processo foi submetido ao processo de liofilização. ECV foi armazenado em recipientes plásticos vedados e mantido sob refrigeração até caracterização e uso na formulação. 


\subsection{Caracterização do extrato}

\subsubsection{Determinação de cor e umidade}

Para a caracterização de cor foi empregado um colorímetro portátil KONICA Minolta-CR400 (Osaka, Japão) com geometria 45/0 e iluminante D65. ECV foi colocado em recipiente de plástico para produto granular (CR-A50) e foi empregado como acessório o tubo de projeção de luz (CR-A33), sendo feita leitura, em triplicata, diretamente na superfície. Foram obtidos os valores de $L^{*}$ (luminosidade), $a^{*}$ (componente vermelho-verde) $\mathrm{e} \mathrm{b}^{*}$ (componente amarelo-azul) e calculada a tonalidade cromática ( $\mathrm{h}=\tan -1$ (b/a).

A umidade de ECV foi determinada em equipamento de infravermelho (OHAUSMB200, EUA) a 105ํํ por 7 min. As medidas foram feitas em triplicata, e o resultado foi utilizado para o cálculo das concentrações em base seca.

\subsubsection{Determinação de bioativos}

Foram empregados solventes de grau cromatográfico: ácido acético (Merck, Darmstadt, Alemanha) e acetonitrila (J.T. Baker, Phillipsburg, EUA) e padrões de ácido-5cafeoilquínico (5-ACQ) e cafeína (Sigma-Aldrich St. Louis, EUA). Água utilizada para preparo das soluções padrões foi obtida através do Purelab Option-Q (Elga, High Wycombe, Reino Unido).

O teor de cafeína e ácidos clorogênicos totais foi determinado conforme Corso et al. (2016). ECV foi dissolvido em solução de ácido acético 5\% (até obter a concentração de $0,1 \mathrm{mg} / \mathrm{mL}$ ) e a solução foi filtrada em membrana de 0,22 $\mu \mathrm{m}$ (Millipore, São Paulo, Brasil).

A análise foi feita em cromatógrafo líquida de alta eficiência (Ultimate 3000, Thermo Scientific, Germering, Alemanha), equipado com um injetor automático de amostras e detector UV-Vis com arranjo de diodos, (Libra S22, Biochrom, Cambridge, Inglaterra), e controlado por software Chromeleon 7.0. Empregou-se coluna Spherisorb ODS-1 (150 x 4,6 mm, $3 \square \mathrm{m}$ ) (Waters, Milford, EUA) e eluição gradiente com solução de ácido acético $5 \%(A)$ e acetonitrila (B) como segue: até $1 \mathrm{~min}, 5 \%$, 6 min 13\% B; vazão de 0,6 mL.min1. A detecção foi feita a $272 \mathrm{~nm}$ para cafeína e $320 \mathrm{~nm}$ para os ácidos clorogênicos. A identificação foi feita com base nos tempos de retenção e espectro no UV. A quantificação foi feita por padronização externa, construindo-se curvas analíticas (6 pontos em triplicata) nas faixas de 3 a $20 \mu \mathrm{g} \cdot \mathrm{mL}-1$ para cafeína $(r=0,9989, p<0,001)$ e 0,5 a 30,0 $\mu \mathrm{g} \cdot \mathrm{mL}-1$ para 
5-ACQ ( $r=0,9997, p<0,001)$. O teor de ácidos clorogênicos totais foi estimado considerando a soma das áreas dos compostos detectados a $320 \mathrm{~nm}$, usando 5-ACQ como padrão, conforme descrito por Corso et al. (2016). Os resultados foram expressos em g de composto por $100 \mathrm{~g}$ de ECV em base seca.

\subsubsection{Análise da atividade antioxidante}

A atividade doadora de íons hidrogênio ao radical ABTS•+ foi avaliada conforme descrito por Vignoli et al. (2011). Uma solução de ABTS (2,2'-azino-bis-(3etilbenzotiazolina-6-ácido sulfônico, pureza $\geq 98 \%$, Sigma Chemical, St. Louis, EUA) foi produzida em meio aquoso reagindo $7 \mathrm{mM}$ de solução estoque ABTS com 2,45 mM de persulfato de potássio (Acros Organics, New Jersey, EUA). A mistura foi armazenada em frasco escuro em temperatura ambiente por $12 \mathrm{~h}$. A solução de ABTS•+ foi diluída com tampão fosfato $(\mathrm{pH} \mathrm{7,4)} \mathrm{até} \mathrm{atingir} \mathrm{absorvância} \mathrm{de} \mathrm{0,700} \mathrm{a} 730 \mathrm{~nm}$. Foi adicionado $10 \mu \mathrm{L}$ de ECV (3 mg/mL) a $4 \mathrm{~mL}$ da solução de ABTS•+ diluída e após 6 minutos de reação foi realizada leitura em espectrofotômetro UV-VIS, Biochrom Libra S22 (Cambridge, Inglaterra), a $730 \mathrm{~nm}$. As análises foram realizadas com medições em triplicata. Soluções de etanol com seis concentrações conhecidas de Trolox (6-hidroxi-2,5,7,8tetrametilchroman-2-ácido carboxílico) (Sigma Aldrich, St. Louis, EUA) na faixa de 0,5 a 8 $\mathrm{mM}$ (em triplicata) foram usadas para a calibração. Os resultados foram expressos como capacidade antioxidante equivalente ao Trolox (TEAC) em g Trolox por $100 \mathrm{~g}$ de amostra em base seca.

\subsection{Preparo das formulações}

Foram realizados testes preliminares para verificar que tipo de formulação cosmética de uso tópico seria compatível com a adição do ECV, sendo: 1) Gel de copolímero do ácido sulfônico acriloildimetiltaurato e vinilpirrolidona neutralizado, 2) Gelcreme de hidroxietilcelulose, 3) Emulsão simples do tipo O/A e 4) Emulsão múltipla do tipo $\mathrm{A} / \mathrm{O} / \mathrm{A}$.

Verificou-se que apenas para a emulsão simples do tipo O/A (ES) e múltipla do tipo A/O/A (EM) foi possível a adição de ECV com formação de emulsões estáveis, sendo então as duas escolhidas para o estudo. Após testes preliminares, foram definidas duas concentrações para adição de ECV: 0,5\% (p/p) e 1,0\% (p/p). As formulações foram 
denominadas: 1) FEM: fórmula base da EM sem adição de extrato, 2) FEM0.5: fórmula contendo ECV a 0,5 \% (p/p), 3) FEM1.0: fórmula contendo ECV a 1,0 \% (p/p), 4) FES: fórmula base da ES sem adição de extrato, 5) FES0.5: fórmula contendo ECV a 0,5 \% (p/p) e 6) FES1.0: fórmula contendo ECV a 1,0\% (p/p). Descrição detalhada dos ingredientes de cada formulação pode ser observada na Tab. 1.

Tabela 1: Composição das seis formulações de de emulsão simples e emulsão múltipla sem extrato (FES e FEM) e adicionadas do extrato de café verde nas proporções de $0,5 \%$ e 1\% (FES0.5, FES1.0, FEM0.5 e FEM1.0).

\begin{tabular}{|c|c|c|c|c|c|c|}
\hline \multicolumn{7}{|c|}{ Formulações $^{*}$} \\
\hline Composição (\%; p/p) & FEM & FEM0.5 & FEM1.0 & FES & FES0.5 & FES1.0 \\
\hline & Qsp & Qsp & Qsp & Qsp & Qsp & Qsp 100.00 \\
\hline & 100.00 & 100.00 & 100.00 & 100.00 & 100.00 & \\
\hline \multicolumn{7}{|l|}{ Água destilada } \\
\hline Cera Polawax ${ }^{\circledR}$ & - & - & - & 15,00 & 15,00 & 15,00 \\
\hline Metilparabeno & 0,20 & 0,20 & 0,20 & 0,20 & 0,20 & 0,20 \\
\hline Propilparabeno. & 0,10 & 0,10 & 0,10 & 0,10 & 0,10 & 0,10 \\
\hline Óleo de Canola & 5,00 & 5,00 & 5,00 & - & - & - \\
\hline $\begin{array}{l}\text { Óleo de rícino } \\
\text { hidrogenado }\end{array}$ & 2,58 & 2,58 & 2,58 & - & - & - \\
\hline $\begin{array}{l}\text { Monooleato de } \\
\text { sorbitano }\end{array}$ & 2,42 & 2,42 & 2,42 & - & - & - \\
\hline $\begin{array}{l}\text { Triglicérides ác. } \\
\text { cáprico/ caprílico }\end{array}$ & - & - & - & 3,00 & 3,00 & 3,00 \\
\hline Ciclometicone & - & - & - & 0,50 & - & - \\
\hline $\mathrm{ECV}^{* *}$ & - & 0,50 & 1,00 & - & 0,50 & 1,00 \\
\hline
\end{tabular}

*FES: formulação emulsão simples sem extrato de café verde (ECV); FES0.5: formulação emulsão simples com 0,5\% ECV; FES1.0: formulação emulsão simples com 1,0\% ECV; FEM: formulação emulsão múltipla sem ECV; FEM0.5: formulação emulsão múltipla com 0,5\% ECV; FEM1.0: formulação emulsão múltipla com $1,0 \%$ ECV.

${ }^{* \star} E C V$ : extrato liofilizado de café verde C. canephora

As formulações FEM foram preparadas por método de emulsificação de inversão de fases, em uma única etapa, usando óleo de canola (Brassica napus L.) obtido da Cocamar (Maringá, Brasil) como emoliente foi utilizado óleo de rícino hidrogenado (BASF, 
Ludwigshafen, Germany) e monooleato de sorbitano (Oxiteno, São Paulo, Brasil) como emulsificantes. Metilparabeno (Synth, São Paulo, Brasil) e propilparabeno (Synth, São Paulo, Brasil) foram utilizados como conservantes. Fase aquosa e oleosa foram aquecidas separadamente a $78 \pm 2 \stackrel{\circ}{ } \mathrm{C}$. A fase aquosa foi lentamente adicionada sobre a fase oleosa sob agitação de 450 rpm (Fisatom 713D São Paulo, Brasil) até $40^{\circ} \mathrm{C}$. Mistura de ECV com água destilada foi lentamente adicionada na EM e agitada por 450 rpm até alcançar a temperatura de $25^{\circ} \mathrm{C}$ (Tab. 1 ).

As formulações FES foram preparada por método de emulsificação de inversão de fases, em duas etapas, contendo cera emulsificante Polawax® (Mapric, São Paulo, Brasil) como emuslificante e espessante, triglicérides do ácido cáprico e caprílico (Mapric, São Paulo, Brasil) e ciclometicone (Mapric, São Paulo, Brasil) como emolientes, metilparabeno (Synth, São Paulo, Brasil) e propilparabeno (Synth, São Paulo, Brasil) como conservantes e água destilada. Fase aquosa e oleosa foram aquecidas separadamente a $75 \pm 2^{\circ} \mathrm{C}$. Fase oleosa foi lentamente adicionada sobre a fase aquosa,

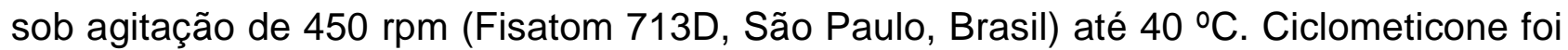
incorporado à emulsão sob agitação. Mistura de ECV com água destilada foi lentamente adicionada na ES e agitada por 450 rpm até alcançar a temperatura de $25^{\circ} \mathrm{C}$.

\subsection{Caracterização das formulações}

\subsubsection{Aspecto morfológicos e organoléptico}

A observação do aspecto foi realizada em dois momentos: macroscopicamente após 24 h em repouso e após o teste de centrifugação.

As formulações $(5 \mathrm{~g})$ foram acondicionadas em tubo de ensaio, cônico, graduado e plástico e foram centrifugadas (Centrífuga Baby I Fanem 206-BL São Paulo, Brasil,) a 3200 rpm por $30 \mathrm{~min}$ à temperatura ambiente, para detectar visíveis modificações ou instabilidades como separação de fases.

Foram observadas as características de aspecto e cor das formulações, as análises foram feitas em triplicata (Brasil, 2007). As amostras foram acondicionadas em vidro relógio e colocadas em fundo branco, sendo verificadas alterações como separação de fase, precipitação e turvação, bem como alteração da cor, uma vez que os extratos possuem 
coloração característica do café.

\subsubsection{Características Físico-Químicas}

$\mathrm{O} \mathrm{pH}$ foi avaliado utilizando o pHmetro digital $\mathrm{pH} 21 \mathrm{pH} / \mathrm{mV}$ (HANNA, Brasil) em temperatura ambiente $\left(25 \pm 5^{\circ} \mathrm{C}\right)$, o eletrodo foi inserido diretamente na amostra (Brasil, 2007).

A densidade foi avaliada utilizando picnômetro de vidro, com capacidade de $10 \mathrm{~mL}$ e temperatura monitorada em $20^{\circ} \mathrm{C}$. A relação entre a massa da amostra e a massa da água representa a densidade específica (Brasil, 2008).

\subsubsection{Avaliação da atividade antioxidante (AA) das formulações}

A análise da atividade AA foi feita por ABTS•+ conforme descrito no item 2.2.3., diferenciando-se apenas no preparo da amostra. As formulações foram diluídas em água $(0,002 \mathrm{~g} / \mathrm{mL})$ e centrifugadas a $25^{\circ} \mathrm{C}(5 \mathrm{~min}, 5000 \mathrm{rpm})$, separando-se apenas a parte aquosa para a análise.

\subsubsection{Análise da viscosidade}

A viscosidade foi analisada conforme recomendado por Brasil (2008), utilizando método da viscosidade dinâmica (Brookfield), adaptando-se o descrito por Prestes et al. (2009). Empregou-se Viscosímetro DV-III ULTRA, banho termostático TC-602 e software Rheocalc V33 (Brookfield, Middleboro, USA). As amostras foram dispostas em recipientes cilíndricos encamisados, com diâmetro de $1,9 \mathrm{~cm}$ e altura de $6,3 \mathrm{~cm}$, para FEM, e diâmetro de $3,7 \mathrm{~cm}$ e altura de $5,0 \mathrm{~cm}$, para FES. Utilizou-se temperatura de análise de $25^{\circ} \mathrm{C}$ e spindles SC4-18 e F96 para FEM e FES, respectivamente.

As velocidades empregadas na análise foram diferentes, bem como os spindles utilizados, considerando que as formulações do tipo FEM e FES apresentam características físico-químicas diferentes, uma vez que, FEM possui características de um líquido (cerca de 1,5 cP), enquanto que FES apresenta características de creme viscoso (na faixa de 31.000 a $72.000 \mathrm{cP}$ ) indicando uma diferença muito grande de viscosidade. Desta forma, 
para análise de FEM foram feitas leituras com a utilização do spindle SC4-18 em 6 diferentes velocidades para as FEM (50, 88, 126, 164, 202 e 240 rpm) e, com o spindle F96 e 7 velocidades para as FES $(0,50,0,75,1,00,1,25,1,50,1,75$ e 2,00 rpm), sendo que cada velocidade foi mantida por 3 min. Para as FEM, os dados da taxa de deformação, taxa de cisalhamento e viscosidade aparente foram coletados após 3 min. Para as FES, os mesmos dados foram coletados em triplicada a cada $45 \mathrm{~s}$, precedidos de $45 \mathrm{~s}$ de estabilização prévia (totalizando $3 \mathrm{~min}$ ), calculando-se a média aritmética das leituras (HÜBL; STEINWENDTNER, 2000 ).

\subsubsection{Avaliação da atividade antioxidante (AA) das formulações}

A análise da atividade $A A$ foi feita por ABTS•+ conforme descrito no item 2.2.3., diferenciando-se apenas no preparo da amostra. As formulações foram diluídas em água $(0,002 \mathrm{~g} / \mathrm{mL})$ e centrifugadas a $25^{\circ} \mathrm{C}(5 \mathrm{~min}, 5000 \mathrm{rpm})$, separando-se apenas a parte aquosa para a análise.

\subsection{Avaliação da estatística}

Os resultados foram comparados por análise de variância (ANOVA), considerando as formulações como causa de variação, e teste de Tukey (nível de significância de 5\%), usando-se o software Statistica 10.0 (Statsoft Inc., Tulsa, USA).

\subsection{RESULTADOS E DISCUSSÃO}

\subsection{Caracterização do ECV}

O extrato de café verde apresentou cor marrom clara, $L^{*}$ de 52 e h de 67 . Marcucci et al. (2013) reportaram para cafés solúveis comerciais brasileiros, regulares e descafeinados, produzidos com diferentes espécies arábica e conilon e processos de secagem (atomizado ou liofilizado), valores de $L^{*}$ de 19,5 a 43,7. O ECV caracterizou-se por uma cor mais clara do que os produtos solúveis de mercado, uma vez que é um extrato de café não torrado e liofilizado.

Quanto à composição em bioativos, ECV apresentou teores de 12,2 g de ACGs/100 g e 3,6 g de cafeína/100 g. Cromatogramas típicos podem ser observados na Fig.1. 

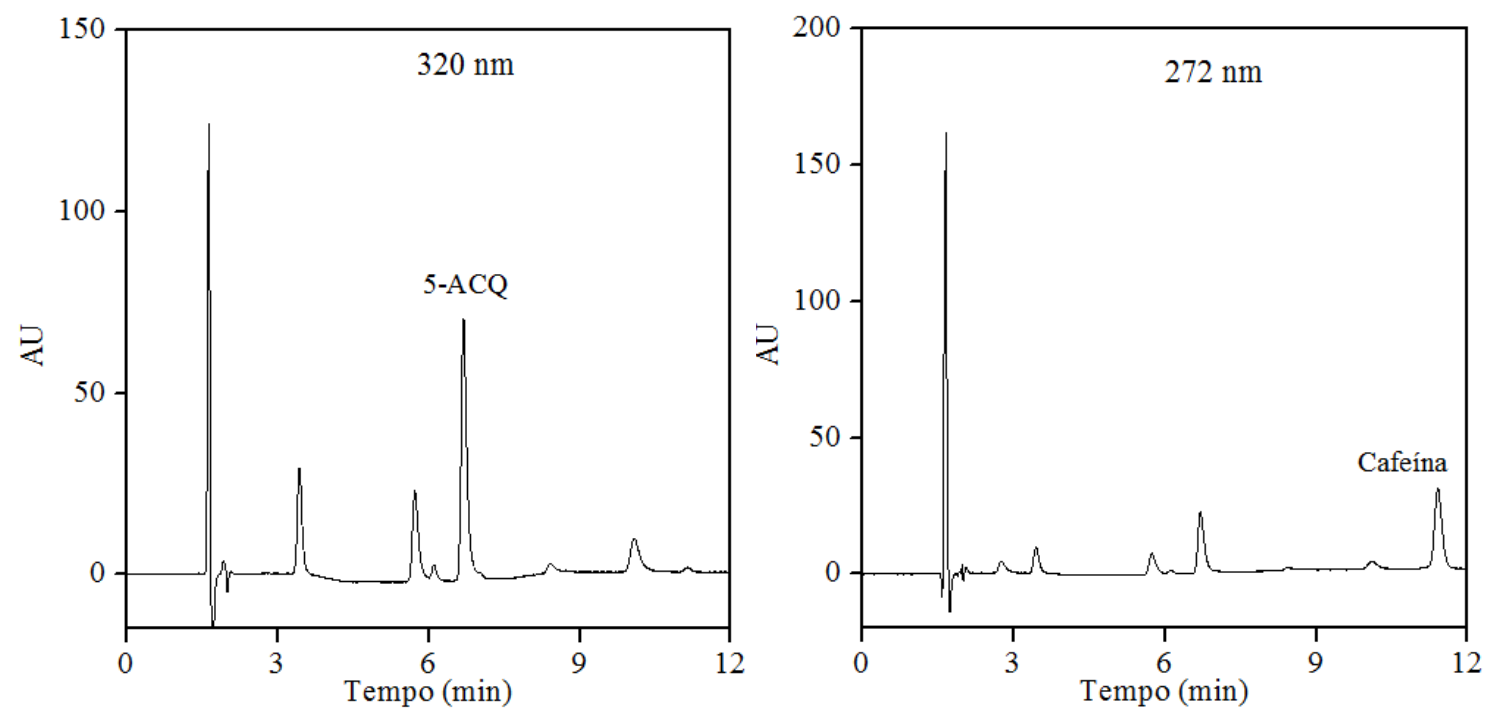

Figura 1: Cromatogramas típicos do extrato de café verde liofilizado (ECV) obtidos a 320 e $272 \mathrm{~nm}$, destacando-se 5-ACQ e cafeína.

Os teores de cafeína do ECV (3,6 g de cafeína/100 g) ficaram dentro da faixa usual para cafés solúveis. Marcucci et al. (2013) reportaram para cafés solúveis comerciais brasileiros teores de cafeína variando de 2,3 a 4,1 g/100g. Vignoli et al. (2011), estudando cafés solúveis liofilizados produzidos com cafés arábica e conilon com diferentes graus de torra e processos de extração, reportaram teores de cafeína de 2,8 a 5,8 g/100g.

Farah e Donangelo (2006) descrevem em sua revisão que café conilon verde apresenta teores de ACG na faixa de 7 a $14 \%$. Corso et al. (2016) relataram teor de ácidos clorogênicos totais variando 2,39 a $5,75 \mathrm{~g} / 100 \mathrm{~g}$ para cafés solúveis liofilizados produzidos com arábica e conilon com dois diferentes graus de torra. Considerando que o ECV foi preparado a partir de café verde e liofilizado, foi observado um alto teor de ácidos clorogênicos (12,2 g de ACGs/100 g) comparados aos cafés solúveis convencionais, que passam pelo processo de torra e podem ter secagem mais agressiva.

A atividade antioxidante do ECV avaliada por ABTS foi de $45,4 \mathrm{~g}$ de Trolox/100 g. Vignoli et al. (2011) relataram valores de ABTS de 18,8 a 36,1 g de Trolox/100 g para cafés solúveis arábica e conilon com diferentes graus de torra e processos de extração. Destacase assim os altos valores de AA do ECV, atribuída a matéria-prima e processo empregados (café $C$. canephora verde e liofilizado) que contribuíram para a manutenção dos bioativos.

\subsection{Caracterização das Formulações}

Com relação ao aspecto morfológico, a formulação emulsão simples (FES) e 
múltipla (FEM), bem como acrescidas dos extratos à 0,5\% (p/p) (FES0.5 e FES1.0) e à 1,0\% (p/p) (FEM0.5 e FEM1.0), após centrifugação por 30 min à temperatura ambiente, não apresentaram separação de fases e nem modificações visíveis, inclusive, mantendo-se estáveis durante $24 \mathrm{~h}$, o que demonstra estabilidade preliminar.

Nos ensaios organolépticos foram verificadas as características de aspecto, cor e odor. Em FEM e FES foi observado coloração branca. Já as formulações acrescidas de ECV, apresentaram cor característica do extrato, sendo que FEM0.5 e FES0.5 apresentaram cor caramelo, e FEM1.0 e FES1.0 mostraram cor café com leite. Quanto ao odor, as amostras contendo ECV apresentaram aroma característico de café torrado, sendo o odor mais intenso quanto maior a concentração de ECV. Apesar do extrato utilizado ser de café verde, com o uso de temperatura no processo de extração, devem ter sido produzidos substâncias voláteis associados ao aroma de café torrado.

A variação de $\mathrm{pH}$ de uma formulação pode modificar as características físicoquímicas do ativo veiculado, influenciando atributos como a sua estabilidade e compatibilidade, comprometendo a segurança e a eficácia da formulação. Os valores do $\mathrm{pH}$ das formulações mantiveram-se entre 4,87 a 5,67 (Tab. 2), sendo compatível com o pH cutâneo $(4,6$ a 5,8), e seguro para o uso. Ressalta-se que ECV possui valor de $\mathrm{pH} 5,10 \pm 0,18$ por conter ACGs e compatível com o pH cutâneo. Não se observou diferença no pH $(p<0,05)$ das FEM com a adição de ECV. A FES apresentou pH 5,67, enquanto FES0.5 e FES1.0 apresentaram 5,04 e 5,03, respectivamente, nesse caso o pH mais ácido, foi atribuído a presença dos ACGs. Foi observado diferença significativa $(p>0,05)$ entre FES0.5 e FEM0.5 e entre FES1.0 e FEM1.0, que se justifica pelo fato de tratar de formulações com composições diferentes. Formulações do tipo FEM apresentaram pH mais ácido $(p>0,05)$ em relação às FES, provavelmente pela estrutura micelar da FEM em promover proteção ao ECV e consequentemente melhorar a atividade de ACG. Formulações contendo extratos vegetais apresentam limitações, uma vez que extratos são facilmente oxidados e susceptíveis à degradação, desta forma, um sistema tipo emulsão múltipla pode melhorar a estabilidade de uma formulação, bem como a eficiência a liberação do extrato.

Os valores de densidade para as formulações de FEM variaram entre 0,98 a 1,00 $\mathrm{g} / \mathrm{cm} 3$, enquanto que as FES a variação foi de 21,07 a 21,31 g/cm3, conforme Tab. 2. A formulação FES apresentou maior densidade $(p>0,05)$ que as adicionadas de extrato (FES0.5 e FES1.0); menor diferença foi observada entre as FES. As formulações FEM apresentaram menor densidade em relação as FES correspondentes, que possuíam maior quantidade de óleos em sua composição. Dados sobre as propriedades físicas de extratos de café também é relevante, uma vez que desfruta de um extenso mercado consumidor,

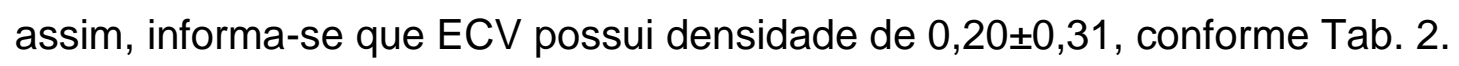




\subsubsection{Atividade antioxidante das formulações}

Resultados mostraram que ECV apresentou boa atividade antioxidante em função de sua composição conter 5-ACQ, cafeína, entre outros compostos, conforme Tab.2. Entretanto, sua adição nas formulações FEM0.5, FEM1.0, FES0.5 e FES1.0 sofreu uma redução, possivelmente, devido a degradação química dos ativos em virtude da composição das formulações. As formulações FES se diferenciaram $(p>0,05)$ quanto à $A A$ (Tab. 2), observando-se aumento da AA com o aumento da concentração de ECV. Nas FEM, adição de ECV também afetou a AA, entretanto, FEM teve menor $(p>0,05)$ AA que FEM0.5 e FEM1.0, mas não houve diferença entre as formulações com ECV (FEM0.5 e FEM1.0). Observou-se assim que para as FES, o aumento na porcentagem de ECV teve maior impacto do que para as FEM.

Todas as formulações FEM apresentaram maiores atividades antioxidantes que as FES correspondentes (Tab. 2). A diferença foi atribuída a presença do óleo de canola nas FEM, que é rico em ácido oléico (55-60\%), possui baixo teor de gordura saturada, boa relação omega-6/omega-3 (2:1) e significativa concentração de ácido linoleico e linolênico. (FARHOOSH, PAZHOUHANMEHR, 2009; WATERHOUSE, WANG, SUN-WATERHOUSE, 2014; LONNI et al., 2015).

Tabela 2: Valores de atividade antioxidante (AA), $\mathrm{pH}$ e da densidade nas seis formulações de emulsão simples e emulsão múltipla sem extrato (FES e FEM) e adicionadas do extrato de café verde nas proporções de 0,5\% e 1\% (FES0.5, FES1.0, FEM0.5 e FEM1.0).

\begin{tabular}{lccc}
\hline \multirow{2}{*}{ Formulações $^{*}$} & \multicolumn{3}{c}{ Parâmetros } \\
\cline { 2 - 4 } & AA (g de Trolox/100 g) & $\mathbf{p H}$ & $\mathbf{d} \mathbf{( g / \mathbf { c m } ^ { 3 } )}$ \\
\hline FES & $0,50^{\mathrm{d}} \pm 0,05$ & $5,67^{\mathrm{a}} \pm 0,06$ & $21,11^{\mathrm{a}} \pm 0,00$ \\
FES0.5 & $2,07^{\mathrm{b}} \pm 0,08$ & $5,04^{\mathrm{b}} \pm 0,06$ & $21,09^{\mathrm{b}} \pm 0,01$ \\
FES1.0 & $3,44^{\mathrm{a}} \pm 0,03$ & $5,03^{\mathrm{b}} \pm 0,03$ & $21,07^{\mathrm{b}} \pm 0,01$ \\
FEM & $1,57^{\mathrm{c}} \pm 0,16$ & $4,92^{\mathrm{c}} \pm 0,03$ & $0,99^{\mathrm{cd}} \pm 0,00$ \\
FEM0.5 & $3,89^{\mathrm{a}} \pm 0,20$ & $4,89^{\mathrm{c}} \pm 0,02$ & $0,99^{\mathrm{c}} \pm 0,00$ \\
FEM1.0 & $4,16^{\mathrm{a}} \pm 0,21$ & $4,87^{\mathrm{c}} \pm 0,01$ & $0,98^{\mathrm{d}} \pm 0,00$
\end{tabular}

Resultados expressos pela média \pm desvio padrão $(n=3)$; médias seguidas de letras minúsculas diferentes na mesma coluna, diferem entre si (Tukey, $p \leq 0,05$ ).

${ }^{*}$ FES: formulação emulsão simples sem extrato de café verde (ECV); FES0.5: formulação emulsão simples com 0,5\% ECV; FES1.0: formulação emulsão simples com 1,0\% ECV; FEM: formulação emulsão múltipla sem ECV; FEM0.5: formulação emulsão múltipla com 0,5\% ECV; FEM1.0: formulação emulsão múltipla com $1,0 \%$ ECV. 


\subsubsection{Análise reológica das formulações}

Observou-se um comportamento Newtoniano para todas as emulsões múltiplas, com e sem ECV (FEM, FEM0.5 e FEM1.0), no qual a viscosidade aparente independe da taxa de cisalhamento (Figura 2A e 2C). No caso das formulações emulsões simples (FES, FES0.5 e FES1.0) foi observado um comportamento não-Newtoniano enquadrado como fluido pseudoplástico, no qual a viscosidade aparente diminui com o aumento da taxa de cisalhamento (Figura 2B e 2D). As emulsões FES foram classificadas como fluidos pseudoplásticos, pois seguem a lei da potência. No reograma fica evidente o comportamento de cada classe de fluidos e no gráfico da viscosidade aparente pela taxa de cisalhamento verifica-se que as formulações FES apresentou maior viscosidade em relação às FEM, pelo fato de sua composição apresentar maior quantidade de óleo e menor de água. Entre as formulações FEM e FES não houve variação em relação às viscosidades aparentes $(p>0,05)$, indicando que a adição do ativo não causou modificações na viscosidade.

Figura 2: Gráfico da viscosidade aparente versus taxa de cisalhamento das formulações emulsão múltipla $(A)$ e formulações emulsão simples $(B)$ e respectivos reogramas (C e D).
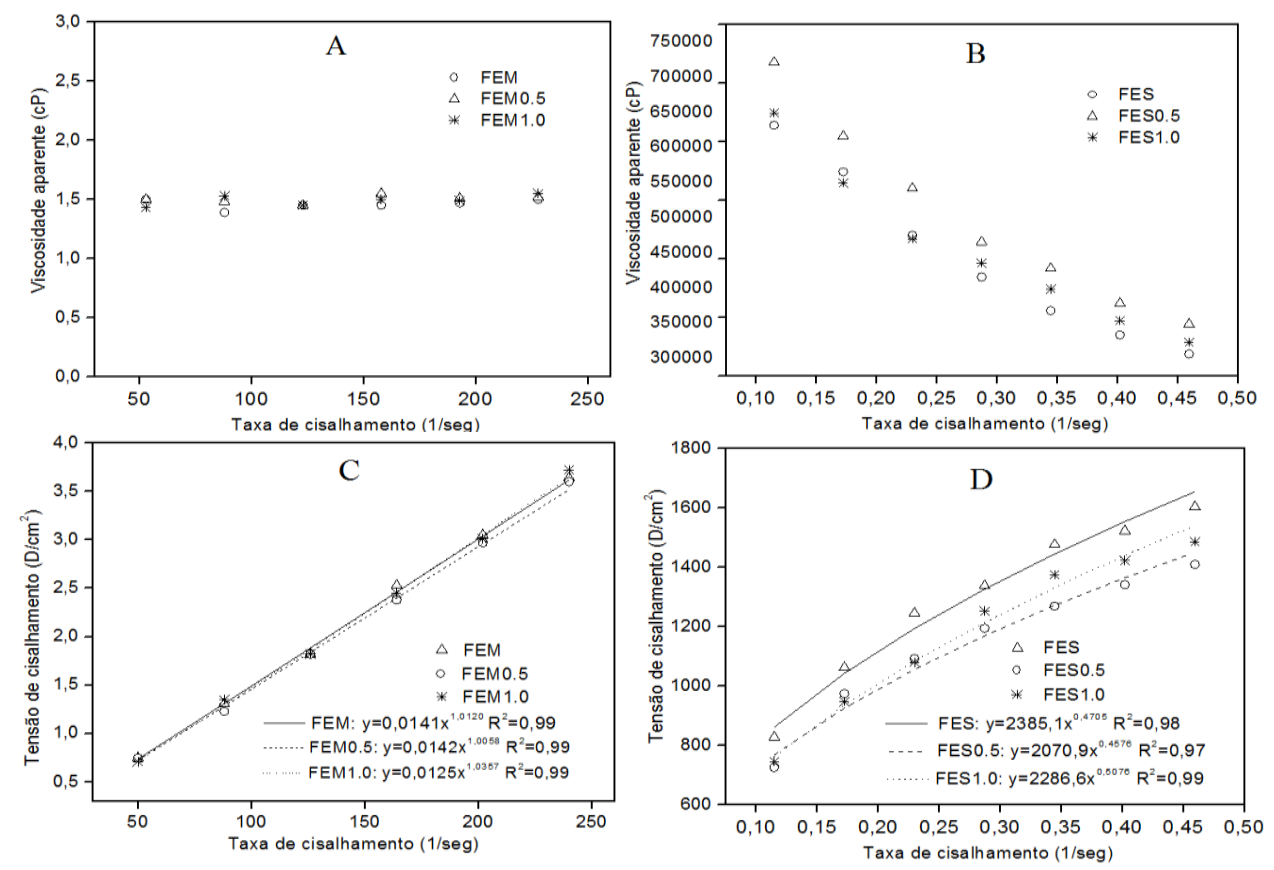

*FES: formulação emulsão simples sem extrato de café verde (ECV); FES0.5: formulação emulsão simples com 0,5\% ECV; FES1.0: formulação emulsão simples com 1,0\% ECV; FEM: formulação emulsão múltipla sem ECV; FEM0.5: formulação emulsão múltipla com 0,5\% ECV; FEM1.0: formulação emulsão múltipla com 1,0\% ECV. 


\section{CONCLUSÃo}

O extrato de café verde Coffea canephora apresentou significante potencial antioxidante, que impactou nas atividades antioxidantes das formulações onde adicionouse ECV. As formulações do tipo emulsão simples e múltipla apresentaram parâmetros organolépticos e físico-químicos compatíveis e com boa viscosidade.

Formulação do tipo emulsão múltipla contendo $0,5 \%$ (p/p) de extrato (FEM0.5) destacou-se pela alta atividade antioxidante com a vantagem de utilizar concentração menor do ECV. Desta forma, sugere-se que FEM0.5 pode ser utilizada na região dos olhos como um potencial preventivo do envelhecimento cutâneo, entretanto, mais estudos são necessários para verificar a sua eficácia e segurança

\section{AGRADECIMENTOS}

Ao CNPq e CAPES pela concessão das bolsas, Universidade Estadual de Londrina e a Universidade Tecnológica Federal do Paraná/Campus Medianeira pelo apoio técnico.

\section{REFERÊNCIAS}

ABIC. Associação Brasileira da Indústria de Café. Exportação Mundial - Principais Países Produtores, 2015. Disponível em: < http://www.abic.com.br/publique/cgi/cgilua.exe/sys/ start.htm >. Acesso em: 26 Abril 2017.

AZULAY, M.M.; LACERDA, C.A.M.; PEREZ, M.A.; FILGUEIRA, A.L.; CUZZI, T.; Vitamina C. Anais brasileiros de dermatologia, 78, n. 3, p. 265-274 2003.

BATISTELA, M.A.; CHORILLI M.; RICCI, G.L. Abordagens no estudo do envelhecimento cutâneo em diferentes etnias. Revista Brasileira de Farmácia, Piracicaba, v. 88, n. 2, p. 5962, 2007.

BRASIL, Ministério da Saúde, Agência Nacional de Vigilância Sanitária. Guia de estabilidade de Produtos Cosméticos. Brasília: ANVISA, 2004. Disponível em acesso em 
BRASIL, Ministério da Saúde, Agência Nacional de Vigilância Sanitária. Guia de Controle de Qualidade de Produtos Cosméticos. Brasília: ANVISA, 2a․ ed., 2008.

CORSO, M.P., VIGNOLI, J.A., BENASSI, M.T. Development of an instant coffee enriched with chlorogenic acids. Journal of Food Science and Technology, v.53, n. 3, p.1380-1388, 2016.

EVANS-JOHNSON, J.A.; GARLICK, J.A.; JOHNSON, E.J.A pilot study of the photoprotective effect of almond phytochemicals in a 3D human skin equivalent. $J$ Photochem Photobiol B 126:17-25, 2013.

FARAH, A.; DONANGELO, C.M. Phenolic compounds in coffee. Brazilian Journal of Plant Physiology, São Paulo, v. 18, n. 1, p. 23-36, 2006.

FARHOOSH, R.; PAZHOUHANMEHR, S. Relative contribution of compositional parameters to the primary and secondary oxidation of canola oil. Food Chemistry v. 114, n. 3, p. 10021006; 2009.

GANCEVICIENE, R.; LIAKOU, A. I.; THEODORIDIS, A.; MAKRANTONAKI, A.; ZOUBOLIS, C. C. Skin anti-aging strategies. Dermato-endocrinology.; v. 4, n. 3, p. 308-319; 2012.

HIRATA, L. L.; SATO, M. E O.; SANTOS, C. A. M. Radicais Livres e o Envelhecimento Cutâneo. Acta Farm. Bonaerense; v. 23, n. 3, p. 418-24, 2004.

HTIBL, J.; STEINWENDTNER, H. Estimation of rheological properties of viscoudebris flow using a belt conveyor. Physics and Chemistry of the Earth, Parte B, v. 25, n. 9, p. 751-5, 2000.

KEDE, M.P.V.; SABATOVICH, O. Dermatologia Estética. Editora Atheneu, 2004.

LONNI, A.; MUNHOZ, V.; LOPES, G.; LONGHINI, R.; BORGHI-PANGONI, F.; DOS SANTOS, R.; JUNQUEIRA, M.; NATALI, M.; LEITE-MELLO, E.; GUIMARAES, F.; BAESSO, M.; SCARMINIO, I.; BRUSCHI, M.; MELLO, J. Development and characterization of multiple emulsions for controlled release of Trichilia catigua (Catuaba) extract. Pharmaceutical Development and Technology, v.21, n. 8, p. 933-942, 2015. 
MARCUCCI, C.T.; BENASSI, M.T.; ALMEIDA, M.B.; NIXDORF, S.L. Teores de trigonelina, ácido 5-cafeoilquínico, cafeína e melanoidinas em cafés solúveis comerciais brasileiros. Química Nova, v. 36, n. 4, p. 544-548, 2013.

NICOLAU, R.A.; COUTO, J.P. Estudo do envelhecimento da derme e da epiderme - revisão bibliográfica. XI Encontro Latino Americano de Iniciação Científica e VII Encontro Latino Americano de Pós-Graduação, p. 2035-2038, 2007.

PRESTES, P.S.; RIGON, R.B.; CORRÊA, N.M.N.; LEONARDI, G.R., Avaliação da estabilidade físico-química de emulsão acrescida de uréia dispersada, ou não, em propilenoglicol. Revista de Ciências Farmacêuticas Básica e Aplicada, v. 30, n. 3, p. 47-53, 2009.

POUILLOT, L.L.; POLLA, P.; TACCHINI, P. Natural antioxidants and their effects on the skin. In: Dayan NK, Romidas L, eds. Formulating, packaging, and marketing of natural cosmetic products, v.1, p .239-258, 2011.

STATSOFT. STATISTICA for Window - Computer programa manual. Versão 10 Tulsa: Statsoft Inc. 2010.

VIGNOLI, J.A.; BASSOLI, D.G.; BENASSI, M.T. Antioxidant activity, polyphenols, caffeine and melanoidins in soluble coffee: The influence of processing conditions and raw material. Food Chemistry, Barking, v. 124, n. 3, p. 863-868, 2011.

VIGNOLI, J.A.; VIEGAS, M.C.; BASSOLI, D.G.; BENASSI, M.T. Roasting process affects differently the bioactive compounds and the antioxidant activity of arabica and conilon coffees. Food Research International, Essex, v. 61, p. 279-285, 2014

VIGNOLI, J.A.; VIEGAS, M.C.; BASSOLI, D.G.; BENASSI, M.T.. VIGNOLI, J. A.; VIEGAS, M. C.; BASSOLI, D. G.; Benassi, M.T. Coffee Brews Preparation: Extraction of Bioactive Compounds and Antioxidant Activity. In: John L. Massey. (Org.). Coffee: Production, Consumption and Health Benefits (Series: Food and Beverage Consumption and Health), v. 1, p. 29-50, 2016. 
WATERHOUSE, G.I.; WANG, W.; SUN-WATERHOUSE, D. Stability of canola oil encapsulated by co-extrusion technology: effect of quercetin addition to alginate shell or oil core. Food Chemistry, v. 142, p. 27-38, 2014. 